\title{
Compare Local Pocket and Global Protein Structure Models by Small Structure Patterns
}

\author{
Xuefeng Cui \\ Computer, Electrical and \\ Mathematical Sciences and \\ Engineering Division \\ King Abdullah University of \\ Science and Technology \\ Thuwal, Saudi Arabia \\ Shuai Cheng $\mathrm{Li}^{*}$ \\ Department of Computer \\ Science \\ City University of Hong Kong \\ Kowloon, Hong Kong
}

\author{
Hiroyuki Kuwahara \\ Computer, Electrical and \\ Mathematical Sciences and \\ Engineering Division \\ King Abdullah University of \\ Science and Technology \\ Thuwal, Saudi Arabia \\ Xin $\mathrm{Gao}^{\dagger}$ \\ Computer, Electrical and \\ Mathematical Sciences and \\ Engineering Division \\ King Abdullah University of \\ Science and Technology \\ Thuwal, Saudi Arabia
}

\begin{abstract}
Researchers proposed several criteria to assess the quality of predicted protein structures because it is one of the essential tasks in the Critical Assessment of Techniques for Protein Structure Prediction (CASP) competitions. Popular criteria include root mean squared deviation (RMSD), MaxSub score, TM-score, GDT-TS and GDT-HA scores. All these criteria require calculation of rigid transformations to superimpose the the predicted protein structure to the native protein structure. Yet, how to obtain the rigid transformations is unknown or with high time complexity, and, hence, heuristic algorithms were proposed.

In this work, we carefully design various small structure patterns, including the ones specifically tuned for local pockets. Such structure patterns are biologically meaningful, and address the issue of relying on a sufficient number of backbone residue fragments for existing methods. We sample the rigid transformations from these small structure patterns; and the optimal superpositions yield by these small structures are refined and reported. As a result, among 11, 669 pairs of predicted and native local protein pocket models
\end{abstract}

\footnotetext{
${ }^{*}$ To whom all correspondence should be addressed (email: shuaicli@cityu.edu.hk).

${ }^{\dagger}$ To whom all correspondence should be addressed (email: xin.gao@kaust.edu.sa).

Permission to make digital or hard copies of all or part of this work for personal or classroom use is granted without fee provided that copies are not made or distributed for profit or commercial advantage and that copies bear this notice and the full citation on the first page. Copyrights for components of this work owned by others than the author(s) must be honored. Abstracting with credit is permitted. To copy otherwise, or republish, to post on servers or to redistribute to lists, requires prior specific permission and/or a fee. Request permissions from permissions@ acm.org. $B C B^{\prime} 15$, September 9-12, 2015, Atlanta, GA, USA

Copyright is held by the owner/author(s). Publication rights licensed to ACM ACM 978-1-4503-3853-0/15/09 ...\$15.00.

http://dx.doi.org/10.1145/2808719.2808756.
}

from the CASP10 dataset, the GDT-TS scores calculated by our method are significantly higher than those calculated by LGA. Moreover, our program is computationally much more efficient.

Source codes and executables are publicly available at http://www.cbrc.kaust.edu.sa/prosta/

\section{Categories and Subject Descriptors}

H.4 [Information Systems Applications]: Miscellaneous; D.2.8 [Software Engineering]: Metrics-complexity measures, performance measures

\section{General Terms}

Protein Pocket, Protein Structure, Optimal Superposition, Quality Assessment of Predicted Protein Structures, Critical Assessment of Techniques for Protein Structure Prediction (CASP)

\section{Keywords}

Protein, Pocket, Structure, Optimal Superposition

\section{INTRODUCTION}

The task of comparing a target protein structure model and its native protein structure model is studied under the protein structure model superposition problem. Formally, the problem is to find the optimal superposition that rotates and translates the target structure model to fit its native structure model according to a scoring function. This is one of the essential tasks in the Critical Assessment of Techniques for Protein Structure Prediction (CASP) competitions [12, 6, 7]. Indeed, the success of the predicted protein structure model assessment process depends mainly on finding the optimal superposition. Moreover, fast and accurate protein structure model superpositions are also widely used for cluster- 
ing millions of predicted decoy models [26, 33] or molecular dynamics simulated models [2].

At this moment, there are several popular scoring functions for evaluating protein structure similarities. The list of popular scoring functions includes root mean squared deviation (RMSD), MaxSub score [21], TM-score [34], GDT-TS and GDT-HA scores [32], and LagSVDi [15]. Unfortunately, methods, to optimize the TM-score and the GDT-TS/GDTHA score, are either unknown or with high time complexity. Thus, heuristic approaches have been adopted in state-ofthe-art methods, TMscore [34] and LGA [31]. Specifically, the heuristics begin by extracting the rigid transformation (a translation and a rotation) from a corresponding residue fragment pair (one from the target model and one from the native model) under the RMSD. The rigid transformation is then applied to the global structures to extract residue pairs which are geometrically close. These pairs are superimposed again under RMSD. This process is repeated until the residue pair set are unchanged. Similar heuristic approaches have also been used in recent protein structure alignment tools $[11,35,20,30,25]$.

It is well known that protein functions are primarily determined by protein structures, but protein functions cannot be determined or predicted reliably by solely global protein structures [28, 24, 19]. Thus, promising alternative methods based on local protein pockets have been proposed for protein function prediction [5, 29, 22] and drug design [27, 18]. These methods are supported by the observation that protein pockets are better conserved than global protein structures for proteins sharing similar functions. Therefore, we propose to evaluate the quality of the predicted models based on local protein pockets. However, the local protein pockets are small (in terms of the number of residues) and highly fragmented. This makes the the protein structure model superposition problem even more challenging for existing methods (e.g., [34, 31]), as they rely on a sufficient number of backbone residue fragments.

In this article, we introduce PROSTA-super, a simple and yet reliable method for solving the protein structure model superposition problem. The method is based on our key observation: there exists a small subset of corresponding residue pairs such that a near-optimal superposition can be determined by minimizing the (weighted) root-mean-square deviation (RMSD) of the corresponding residue pairs. Because of the simplicity of our protein structure model superposition method, it is called PROSTA-super, where the word "prosta" means simple in Polish and the word "super" hints the superposition problem to be solved. Variety kinds of scoring functions, such as the widely used TM-score [34], GDT-TS and GDT-HA scores [32], can be adopted in our method. However, finding the best scoring function is out of the scope of this study.

Comparing to existing protein structure model superposition methods (e.g., [34, 31]), the method is specifically tuned for optimally superimposing protein pocket models. To develop a method that focuses on the challenging protein pocket superposition, we followed the observation that protein pockets tend to be highly fragmented. This led us to believe that locally related residues defined on residueresidue contacts, rather than those defined on consecutive backbone fragments, are more informative in such cases. Thus, PROSTA-super considers residue contact cliques during the initial superposition sampling process. Since protein pockets tend to be small and the contact clique size is also small in practice, the computational time of the clique finding is fast and manageable. Moreover, two contact cliques are used together to find similarities between not only local contact groups but also global topologies. The general protein structure model superposition could also be benefited from these improvements.

To evaluate the performance of PROSTA-super, we compared it with LGA [31] using 11,669 pairs of predicted and native local protein pocket models and 26, 135 pairs of predicted and native global protein structure models from the CASP10 dataset [12]. We found that the GDT-TS scores [32] calculated by PROSTA-super were significantly higher than those calculated by LGA for the protein pocket models. Even for the global protein structure models, which PROSTA-super was not specifically designed for, we found that the GDT-TS scores were slightly better than those of LGA. Also, comparing the computational time of the two methods, we found that PROSTA-super was computationally much more efficient. These demonstrate the usefulness and reliability of our new method when it comes to local protein pocket model superpositions as well as global protein structure model superpositions.

\section{METHODS}

For the protein structure model superposition problem, a target protein structure model is optimally superimposed to its native protein structure model. Since the target model and its native model are two models of the same protein, there is a one-to-one relashionship between the residues from the target model and those from the native model. Specifically, two residues, one from the target model and one from the native model, are corresponded if and only if they have the same residue indices (defined by the PDB format [1]). Thus, we simply call such a residue pair as a corresponding residue pair in this article.

Our protein structure model superposition method can be divided into three steps. In the first step, the initial superpositions are sampled by minimizing the RMSD of small pre-defined subsets of corresponding residue pairs. Then, for each sampled superposition, the respective rigid transformation is applied to the two structures and the alignment score is calculated to evaluate the quality of the superposition. In the second step, the superpositions with relatively high alignment scores are iteratively refined by resampling superpositions that minimizes the weighted RMSDs of small random subsets of corresponding residue pairs. In the final step, a subset of selected superpositions are iteratively refined again by resampling superpositions that minimizes the weighted RMSDs of (potentially) large subsets of similar corresponding residue pairs. As a result, the superposition yielding the highest alignment score is reported as the optimal one. These three steps are further explained in details in the following three subsections.

\subsection{Initial Superposition Sampling}

In the first step, the initial superpositions are sampled with small subsets of corresponding residue pairs. One corresponding residue pair is simply represented by the residue index. For the sake of simplicity, let $2 N$ be the number of residue pairs in a subset (some subsets are formed by two groups of $N$ residue pairs), and $\left\{S_{1}, S_{2}, \ldots, S_{2 N}\right\}$ represent the residue indices within the subset. Moreover, let 
$C\left(S_{i}, S_{j}\right)=1$ if two residue pairs $S_{i}$ and $S_{j}$ are contacted (including the case that $\left.S_{i}=S_{j}\right)$, and $C\left(S_{i}, S_{j}\right)=0$ otherwise. If $C\left(S_{i}, S_{j}\right)=1$ for all $i, j \in\left\{S_{1}, S_{2}, \ldots, S_{N}\right\}$ and $i, j \in$ $\left\{S_{N+1}, S_{N+2}, \ldots, S_{2 N}\right\}$, contact groups $\left\{S_{1}, S_{2}, \ldots, S_{N}\right\}$ and $\left\{S_{N+1}, S_{N+2}, \ldots, S_{2 N}\right\}$ are called contact cliques of size $N$. Here, any common contact definition (e.g., based on atomatom distances [8] or contact areas [17]) can be adopted. Using above notations, six kinds of small subsets are defined:

Local Consecutive Subset (LCON): a subset of corresponding residue pairs that are consecutive (connected by peptide bonds) on the protein backbone. Formally, the subset is a local consecutive subset if and only if the residue indices satisfy that $S_{i+1}-S_{i}=1$ for $i \in\{1,2, \ldots, 2 N-1\}$.

Remote Consecutive Subset (RCON): a subset of corresponding residue pairs that combines two local consecutive subsets of $N$ residue pairs. Formally, the subset is a remote consecutive subset if and only if the residue indices satisfy that $S_{N+1}-S_{N}>1$ and $S_{i+1}-$ $S_{i}=1$ for $i \in\{1,2, \ldots, N-1\} \cup\{N+1, N+2, \ldots, 2 N-1\}$.

Local Gapped Subset (LGAP): a subset of corresponding residue pairs that contain gaps of a small constant size (i.e., a small constant number of residues) on the protein backbone. Formally, the subset is a local gapped subset if and only if the residue indices satisfy that $S_{i+1}-S_{i}=g$ for $i \in\{1,2, \ldots, 2 N-1\}$ and $1<g<g_{m}$. Here, $g_{m}$ is employed to make sure that the subset is modeling a local structure pattern, and we simply set $g_{m}=2 N$ in this study. Certainly, $g_{m}$ can be set to other values or even infinity.

Remote Gapped Subset (RGAP): a subset of corresponding residue pairs that combines two local gapped subsets of $N$ residue pairs. Formally, the subset is a remote gapped subset if and only if the residue indices satisfy that $S_{N+1}-S_{N}>g$ and $S_{i+1}-S_{i}=g$ for $i \in\{1,2, \ldots, N-1\} \cup\{N+1, N+2, \ldots, 2 N-1\}$ and $1<g<g_{m}$.

Local Clique Subset (LCLI): a subset of corresponding residue pairs that contains two contact cliques of size $N$ and there exists at least one contact between the two contact cliques. Formally, the subset is a local clique subset if and only if the residue indices satisfy that $\left\{S_{1}, S_{2}, \ldots, S_{N}\right\}$ and $\left\{S_{N+1}, S_{N+2}, \ldots, S_{2 N}\right\}$ are two contact cliques of size $N$ and $\exists i \in\{1,2, \ldots, N\}$ and $j \in$ $\{N+1, N+2, \ldots, 2 N\}$ such that $C\left(S_{i}, S_{j}\right)=1$.

Remote Clique Subset (RCLI): a subset of corresponding residue pairs that contains two contact cliques of size $N$ and there does not exist any contacts between the two contact cliques. Formally, the subset is a remote clique subset if and only if the residue indices satisfy that $\left\{S_{1}, S_{2}, \ldots, S_{N}\right\}$ and $\left\{S_{N+1}, S_{N+2}, \ldots, S_{2 N}\right\}$ are two contact cliques of size $N$ and $\nexists i \in\{1,2, \ldots, N\}$ and $j \in\{N+1, N+2, \ldots, 2 N\}$ such that $C\left(S_{i}, S_{j}\right)=1$.

Each type of the above subsets captures its own strengths over others. The consecutive subsets are the classic subsets that have been widely used for the protein structure alignment problem [35, 25] and the protein structure model superposition problem $[34,31]$. One main reason behind the popularity of the consecutive subsets is their simplicity and fast speed. The new gapped subsets have the advantage of increasing the backbone coverage without increasing the size of the subsets. The increased backbone coverage might potentially be more robust to poor local protein qualities (i.e., noisy atom coordinates). The new clique subsets are more biologically meaningful because they are defined on residue-residue contacts that are not limited to backbone peptide bonds. Thus, the consecutive subsets (that are defined on backbone peptide bonds) tend to be special cases of the clique subsets. While the local subsets tend to model local shapes of the backbone, the remote subsets tend to model the global topology of the backbone. It can be shown that remote topology similarities are more robust to less similar protein structure models with little local structure similarities $[3,4]$.

To use PROSTA-super, one can specify which types of subsets to be used, and the initial superpositions are sampled from those subsets of corresponding residue pairs as followings. First, for each subset that satisfies one of the specified subset definitions, the superposition that minimizes the RMSD of the corresponding residue pairs is calculated (by the well-known Kabsch algorithm [9] or a quaternion-based characteristic polynomial [23]). Then, the superposition is applied to the protein structure models, and the quality of the superposition is evaluated by an existing scoring function, such as TM-score [34], GDT-TS and GDT-HA scores [32]. Finally, the superpositions are sorted by the calculated scores, and the highly scored ones are selected to be refined in the next step. In our current implementation, $L$ highly scored superpositions are selected, where $L$ is the number of corresponding residue pairs of the input protein structure models.

\subsection{Superposition Refinement with Random Subsets}

In the second step, the promising superpositions with relatively high alignment scores are iteratively refined with small random subsets of corresponding residue pairs. Specifically, in each iteration, a random subset of $2 N$ corresponding residues pairs are selected for each promising superposition. For each random subset, the superposition that minimizes the weighted RMSDs of the corresponding residue pairs is calculated by a quaternion-based characteristic polynomial [23]. Then, similar to the previous step, the superposition is applied to the protein structure models and the quality of the superposition is evaluated. If a higher quality superposition is found, it is used to replace the old promising superposition for the next iteration. Here, it might be worth noting that the size of the random subset remains consistent to that of the subsets defined in the first step, Also note that the number of iterations can be specified by the end user.

There are two major differences between this refinement step and the first initial superposition sampling step. First, instead of scanning some specific types of subsets, randomly selected subsets are used for the refinement. Second, instead of optimizing the classic RMSD, the weighted RMSD is used as the objective function. Specifically, weight $w_{i}$ of the $i$ th corresponding residue pair is set to its TM-score [34] as following:

$$
w_{i}=\frac{1}{1+d_{i}^{2} / d_{0}^{2}}
$$


where $d_{i}$ is the distance between the $i$-th superimposed residue pair from the previous iteration, and $d_{0}=1.24\left(L_{r}-15\right)^{1 / 3}-$ 1.8. Thus, minimizing the TM-score weighted RMSD tends to minimize the TM-score more effectively and to converge more efficiently [30]. It might be worth mentioning that the weight calculation requires a superposition as the input, and thus it cannot be adopted in the first step.

\subsection{Superposition Refinement with Similar Subsets}

In the last step, the promising superpositions are further iteratively refined with (potentially) large subsets of similar corresponding residue pairs. Similar to the previous refinement step, a subset of corresponding residue pairs are selected for each promising superposition in each iteration. For each selected subset, the superposition that minimizes the weighted RMSDs of the corresponding residue pairs is calculated, and a higher quality superposition is kept as the new promising superposition (if found). Thus, the main advantage of minimizing a weighted RMSD that is more correlated to minimizing the ultimate alignment scoring function is inherited from the previous refinement step. Finally, the superposition yielding the highest alignment score is reported as the optimal one in this step.

Unlike the previous refinement step that randomly selects subsets of $2 N$ corresponding residues pairs, new potentially large subsets of similar corresponding residue pairs are selected in this final refinement step. Specifically, all similar corresponding residue pairs within a distance threshold (or above a similarity threshold) are selected. Moreover, the distance (or similarity) threshold for selecting residue pairs is increased (or decreased) slightly after each iteration. Thus, there are no constraints on the size of the selected subsets. In our current implementation, a TM-score [34] threshold is used for each iteration, and the threshold is updated from one (identical residue pairs) towards zero (unrelated residue pairs) with a constant step of 0.03 after each iteration.

\section{SUPERIMPOSING PROTEIN STRUCTURE MODELS}

In the tenth Critical Assessment of Techniques for Protein Structure Prediction (CASP10) [12], each predicted protein structure model was optimally superimposed to the native protein structure model, and the quality of the predicted model was measured by the alignment score calculated from the superimposed models. Totally, 103 target protein structures are predicted by servers (i.e., without human interactions), and 26, 135 pairs of predicted and native protein structure models (with at least 20 corresponding residue pairs) are conducted. For each pair of predicted and native models conducted from CASP10, the GDT-TS scores [32] are calculated using our method and state-of-the-art LGA method [31]. Previously, the GDT-TS score and the LGA method have been used by CASP competitions since $1998[32,16,10,12]$. In this study, the GDT-TS scores are normalized by the number of corresponding residue pairs between predicted and native models, and $N$ is set to be the smallest meaningful value of 3 .

\subsection{Performance Analysis after Step 1}

First, we evaluate the suitability of each type of subsets of corresponding residue pairs defined in Section 2.1 and to check if some particular types of subsets perform better in some particular situations. This is done by calculating and comparing the GDT-TS scores [32] of the initial superpositions sampled from different types of subsets, as shown in Figure 1. For this analysis, no refinements are applied on any initial superpositions.

From Figures 1(a), 1(b) and 1(c), it can be seen that the alignment scores calculated by remote subsets tend to be higher than those calculated by local subsets. This is especially true for consecutive subsets shown in Figure 1(a), where $47.56 \%$ of the alignment scores are at least 0.03 higher if remote consecutive subsets are used instead of local consecutive subsets. The numbers are reduced to $5.38 \%$ and $0.52 \%$ for gapped subsets and clique subsets, respectively. In the best cases, the alignment scores are at least 0.1 higher if remote subsets are used instead of local subsets. Moreover, it is rare (i.e., with a probability less than $0.2 \%$ ) to see cases where local subsets outperform remote subsets. Indeed, such cases do not even exist for consecutive subsets. Therefore, for accuracy sensitive tasks such as the predicted protein structure model evaluation of CASP competitions [12], remote subsets are more suitable for the task.

For local subsets, it is well demonstrated that local clique subsets outperform local gapped subsets, which outperform local consecutive subsets. Specifically, Figure 1(e) shows that $5.12 \%$ of the alignment scores are at least 0.03 higher if local clique subsets are used instead of local gapped subsets, and Figure $1(\mathrm{~d})$ shows that $27.95 \%$ of the alignment scores are at least 0.03 higher if local gapped subsets are used instead of local consecutive subsets. Since local consecutive subsets are special cases of local clique subsets, local clique subsets are guaranteed to outperform local consecutive subsets. As a result, $59.27 \%$ of the alignment scores are at least 0.03 higher if local clique subsets are used instead of local consecutive subsets, as shown in Figure 1(f). Therefore, although local consecutive subsets are the most widely used local subsets, they are the worst performed local subsets and they should be replaced by local gapped subsets or local clique subsets for better performance.

For remote subsets, the situation becomes more complicated as shown in Figure 1(g). Although remote gapped subsets are more likely to produce higher alignment scores, the biggest improvements are credited to remote consecutive subsets. Similarly, although remote clique subsets are more likely to produce higher alignment scores, the biggest improvements are credited to remote consecutive subsets, as shown in Figure 1(i). Moreover, there is no strong evidence supporting either remote gapped subsets or remote clique subsets perform better in Figure 1(h). Indeed, we observed that, for proteins with more than 50 residues, all three types of remote subsets perform similarly. Specifically, remote clique subsets slightly outperform remote gapped subsets, which slightly outperform remote consecutive subsets.

We found that the performance uncertainty for remote subsets comes from short proteins (i.e., with less than 50 residues). For short proteins, the number of subsets satisfying the definitions of remote gapped and clique subsets are reduced significantly. For remote gapped subsets, this is because that a remote gapped subset could cover residue indices between $i$ and $i+g \times(2 N-2)+k(k>g>1)$, which involves at least $4 N$ residues on short protein backbones. For remote clique subsets, this is because that fewer contact cliques would have no contacts with other contact cliques in 


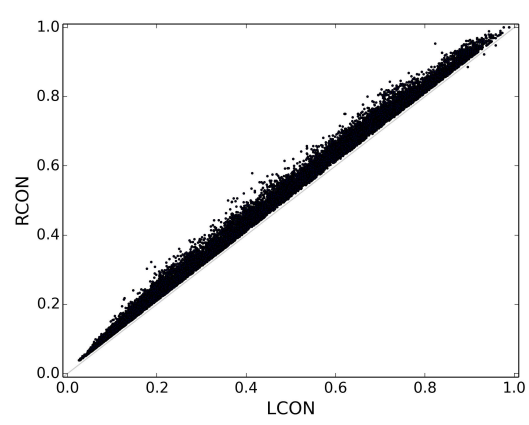

(a) LCON v.s. RCON

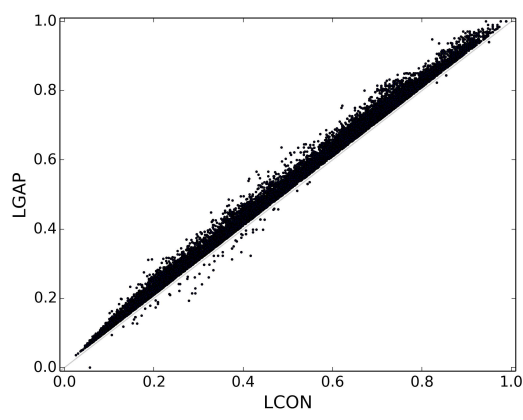

(d) LCON v.s. LGAP

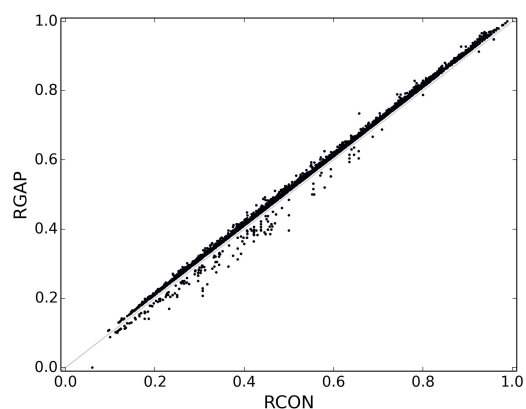

(g) RCON v.s. RGAP

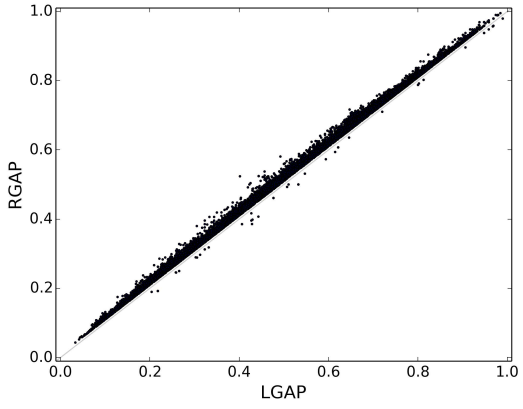

(b) LGAP v.s. RGAP

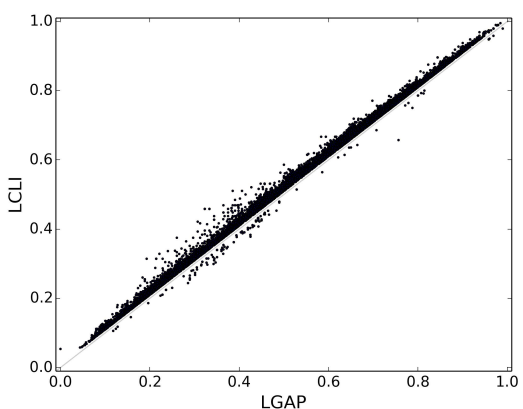

(e) LGAP v.s. LCLI

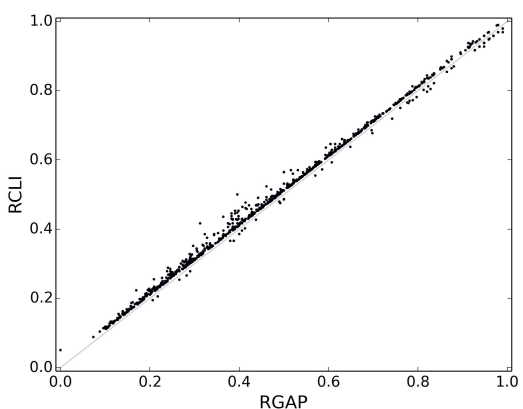

(h) RGAP v.s. RCLI

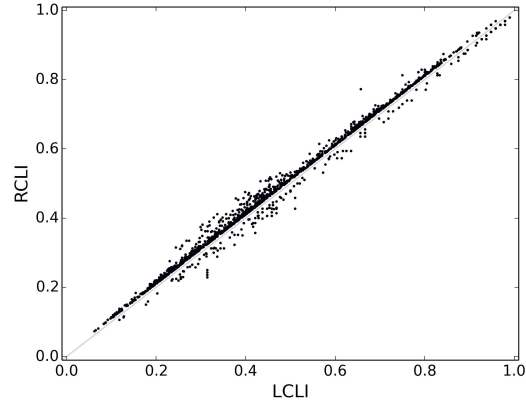

(c) LCLI v.s. RCLI

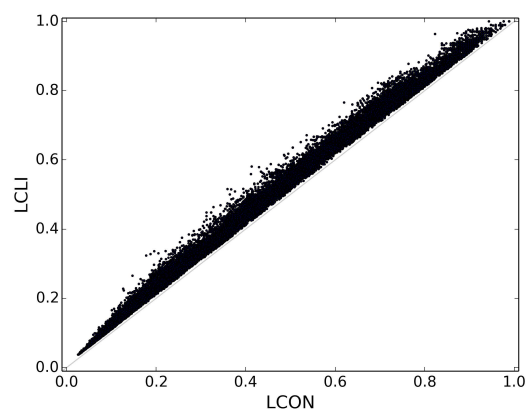

(f) LCON v.s. LCLI

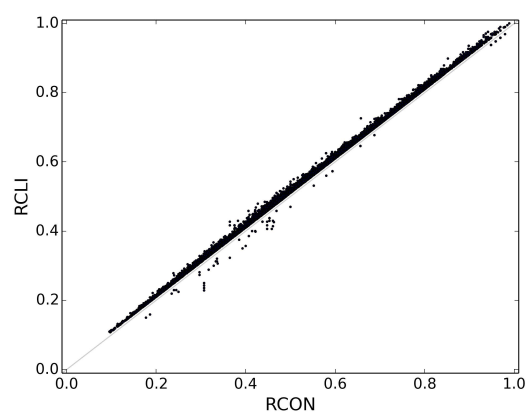

(i) RCON v.s. RCLI

Figure 1: Comparisons of the GDT-TS scores calculated by the initial superposition sampling with the six types of subsets of corresponding residue pairs defined in Section 2.1 (only cases with alignment score differences over 0.01 are shown): (a-c) for all types of subsets, remote subsets tend to outperform local subsets; (d-f) for the three types of local subsets, local clique subsets tend to outperform local gapped subsets, which tend to outperform local consecutive subsets; (g-i) for proteins with more than 50 residues, all three types of remote subsets tend to perform similarly; and for proteins with less than 50 residues, remote consecutive subsets tend to outperform remote consecutive and gapped subsets because of the relatively small reduction on the number of subsets satisfying the remote consecutive subset definition.

short proteins. Although the number of remote consecutive subsets is also reduced, it should be the least reduced one. Therefore, due to the reduced numbers of remote subsets in short proteins, the performance becomes less predictable for remote gapped and clique subsets, while remote consecutive subsets tend to perform more reliably.

\subsection{Performance Analysis after Step 2}

Next, we evaluate the performance of our refinement method with randomly selected subsets of corresponding residue pairs, as described in Section 2.2. Recall that there are six types of subsets defined for the initial superposition sampling step.
In this experiment, only local and remote gapped subsets are used to produce promising superpositions to be refined, and this should be fine because the focus of this experiment is on the random subset refinement method. Then, the GDTTS scores [32] after zero, 10, 25, 50, 100 and 200 iterations of the random subset refinements have been calculated and compared. Here, we stop refinements after 200 iterations because the computational time required for 200 iterations is approximately the same as that required for the LGA method [31]. It might be worth mentioning that the final refinement step with larger subsets of similar corresponding residue pairs are not applied in this experiment. 


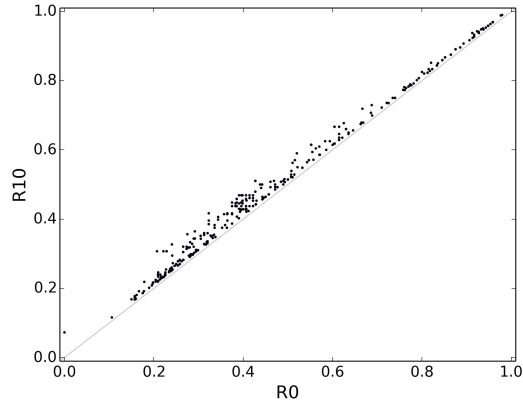

(a) Zero v.s. 10 iterations

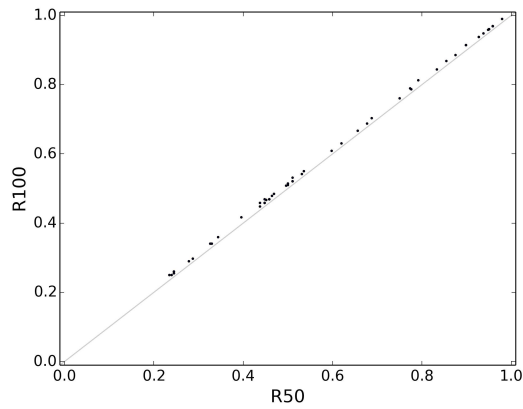

(d) 50 v.s. 100 iterations

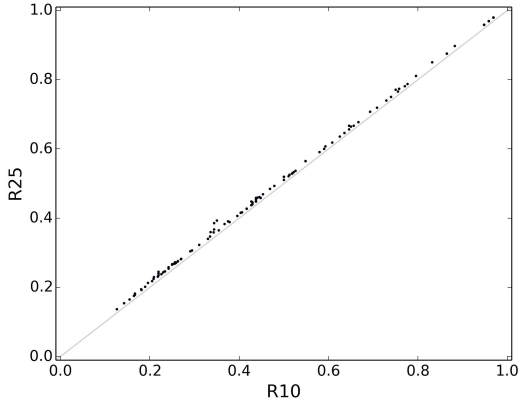

(b) 10 v.s. 25 iterations

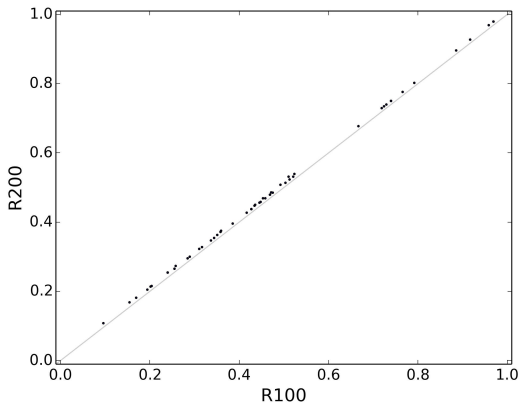

(e) 100 v.s. 200 iterations

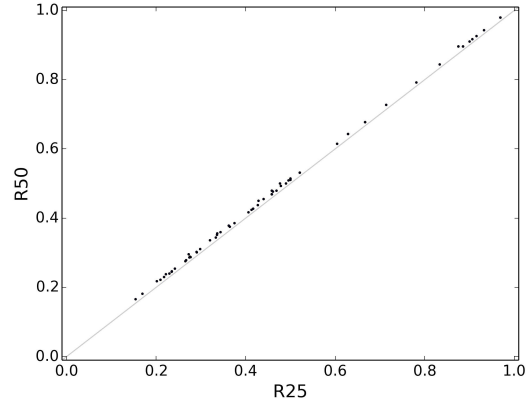

(c) 25 v.s. 50 iterations

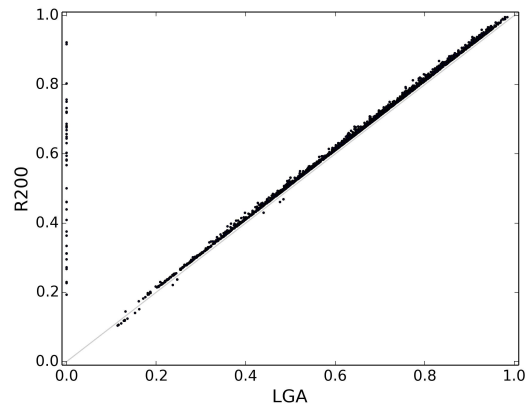

(f) LGA v.s. 200 iterations

Figure 2: Comparisons of the GDT-TS scores calculated after zero, 10, 25, 50, 100 and 200 iterations of the random subset refinements defined in Section 2.2 (only cases with alignment score differences over 0.01 are shown): (a) the biggest alignment score improvements come from the first few refinement iterations; (b-e) as exponentially more refinement iterations are applied after 50 iterations, the number of cases that the alignment scores are noticeably improved (i.e., by at least 0.01) is only decreased slightly; (f) after 200 refinement iterations, the alignment score calculated by our method is at most 0.016 lower than that calculated by LGA; and all these observations support that a near-optimal superposition can be determined by minimizing the weighted RMSD of a small subset of corresponding residue pairs.

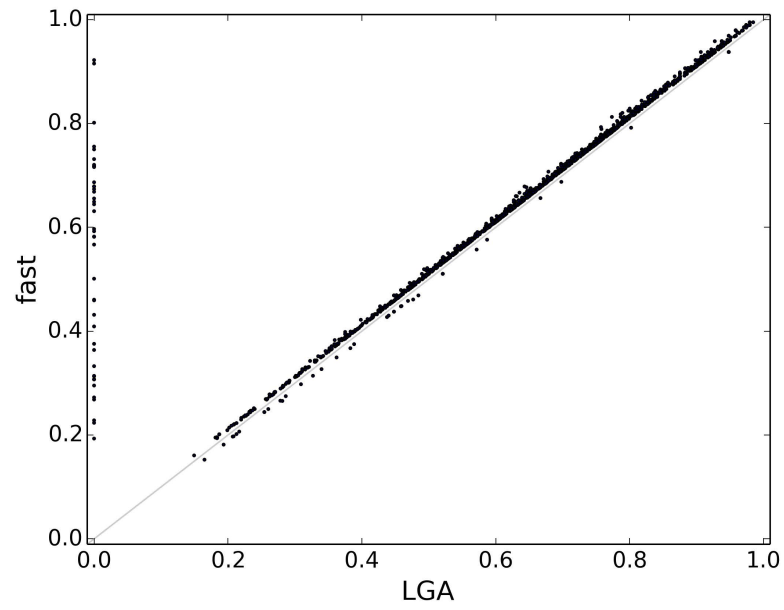

(a) LGA v.s. PROSTA-super (fast version)

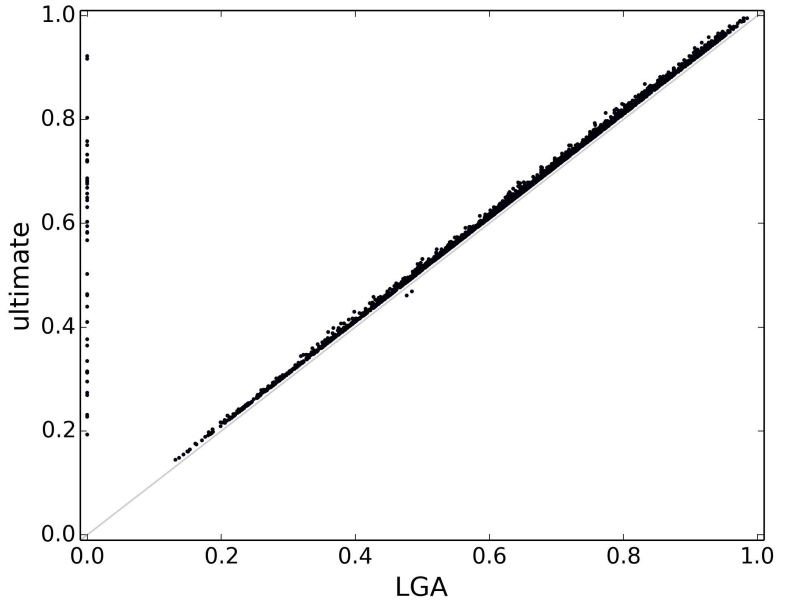

(b) LGA v.s. PROSTA-super (ultimate version)

Figure 3: Comparisons of the GDT-TS scores calculated by PROSTA-super and LGA (only cases with alignment score differences over 0.01 are shown): (a) for the fast version of PROSTA-super, there are 1,989 cases that the alignment scores calculated by PROSTA-super are at least 0.01 higher; the t-value of 150.99 of paired alignment scores calculated by PROSTA-super and LGA indicates that PROSTA-super produces significantly higher alignment scores than LGA; (b) for the ultimate version of PROSTA-super, there are 3, 969 cases that the alignment scores calculated by PROSTA-super are at least 0.01 higher; and the increased t-value of 220.30 indicates that the alignment scores can be even further improved by PROSTA-super. 
The importance of applying the random subset refinements is well demonstrated in Figure 2(a). Totally, after only 10 refinement iterations, there are 112 cases that the alignment scores are improved by at least 0.03 and 340 cases that the alignment scores are improved by at least 0.01 . In the best case, the alignment score is improved by 0.1 after refinements. Therefore, even if refinements are repeated for only a small number of iterations, the improvements could be noticeable and even significant. Actually, the biggest alignment score improvements come from the first few refinement iterations, and it is becoming harder and harder to refine the superposition as more and more refinement iterations have been applied, as demonstrated next.

While more and more random subset refinement iterations have been applied, the alignment scores keep improving as shown in Figures 2(b), 2(c), 2(d) and 2(e). However, after 25 refinement iterations, there are no observed cases that the alignment scores can be improved by at least 0.03. Moreover, the number of cases that the alignment scores are improved by at least 0.01 is decreased by approximately three folds (i.e., from 340 to 128 ) after 25 refinement iterations, and then by another approximately two folds (i.e., from 128 to $65)$ after 50 refinement iterations. As exponentially more refinement iterations are applied after 50 refinement iterations, the number of such cases only decreases slightly. Therefore, the results suggest to apply 50 refinement iterations in order to balance the accuracy and the speed. Certainly, one can trade accuracy for speed or vice versa by changing the number of refinement iterations.

Recall that our method is designed based on the key observation that there exists a small subset of corresponding residue pairs such that a near-optimal superposition can be determined by minimizing the weighted RMSD of the corresponding residue pairs. As LGA [31] has been the reference method to calculate GDT-TS scores [32] in previous CASP competitions $[32,16,10,12]$, we compared the GDT-TS scores calculated by our method and those calculated by LGA in Figure 2(f). Since the final refinement step based on large subsets are not applied in this experiment, only small subsets of size 6 have been used in our method here. It is observed that, in the worst case, the alignment score calculated by our method is only 0.016 lower than that calculated by LGA. Therefore, if the GDT-TS scores calculated by LGA are presumed to be near-optimal, our observation supports that a near-optimal superposition can be determined by minimizing the weighted RMSD of a small subset of corresponding residue pairs.

\subsection{Performance Analysis after Step 3}

Finally, we evaluate the performance of the full version of our protein structure model superposition method, called PROSTA-super. Then, the GDT-TS scores [32] calculated by our PROSTA-super method are compared with those calculated by the LGA method [31]. Since similar refinement methods have been well studied in the protein structure model superposition problem [31, 34], the performance of our final large similar subset refinement step, introduced in in Section 2.3, is not included in this article. In summary, our results show that the large similar subset refinement is highly efficient with low computational cost.

In this experiment, two variants of our PROSTA-super method are evaluated by specifying two different settings. For the fast version, local and remote gapped subsets are employed in the initial superposition sampling step, and 50 refinement iterations are employed in the random subset refinement step. For the ultimate version, local gapped subsets, local clique subsets, remote consecutive subsets and remote gapped subsets are employed in the initial superposition sampling step, and 200 refinement iterations are employed in the random subset refinement step. Moreover, the final refinement method with large subset of similar corresponding residue pairs is also employed in this experiment. Certainly, other settings can be specified by end users to specifically tune our PROSTA-super method.

The fast version of our PROSTA-super method has already achieved higher alignment scores with faster speed than LGA [31]. From Figure 3(a), it can be seen that there are 1,989 cases that the alignment scores calculated by PROSTA-super are at least 0.01 higher, while there are 39 cases that the alignment scores calculated by LGA are at least 0.01 higher. This includes 42 cases that LGA refused to report alignment scores because of atom clashes. If the alignment scores calculated by PROSTA-super and LGA (excluding cases that LGA refused to report alignment scores) are treated as paired samples, the paired t-test [36] can be performed to evaluate the significance of the alignment score improvements. As a result, a t-value of 150.99 and a two-tailed p-value of zero (due to numerical limitations) are obtained. Therefore, the alignment scores calculated by PROSTA-super are significantly higher than those calculated by LGA while the computational time is reduced by approximately half.

The ultimate version of our PROSTA-super method achieved even higher alignment scores as shown in Figure 3(b). Specifically, there are 3,969 cases that the alignment scores calculated by PROSTA-super are at least 0.01 higher, while there are only two cases that the alignment scores calculated by LGA [31] are at least 0.01 higher. Other than the 42 cases that LGA refused to report alignment scores, the alignment scores are improved by up-to 0.039 and 0.016 for PROSTA-super and LGA, respectively. The t-test on the cases that LGA reports alignment scores yields a t-value of 220.30 and a two-tailed p-value of zero (due to numerical limitations). Therefore, the alignment scores calculated by PROSTA-super can be further improved over LGA while the computational time is increased by approximately three folds.

\section{SUPERIMPOSING PROTEIN POCKET MODELS}

In this experiment, we evaluated the performance of our protein structure model superposition method on protein pockets, and compared the GDT-TS scores [32] calculated by our method to those calculated by LGA [31]. Generally, a protein pocket can be defined by either its geometric shape [14] or its interaction energy [13]. Moreover, it has been shown that the largest pocket of an enzyme tends to carry the function of the enzyme [5]. Thus, we used the widely used geometric-based CASTp program [14] to calculate the largest pocket for each native target protein structure of CASP10 [12]. As a result, there are 48 protein pockets with at least 20 residues and 11,669 pairs of predicted and native protein pocket models for this analysis. From Figure 4, it can be seen that most pockets contain less than 50 residues 


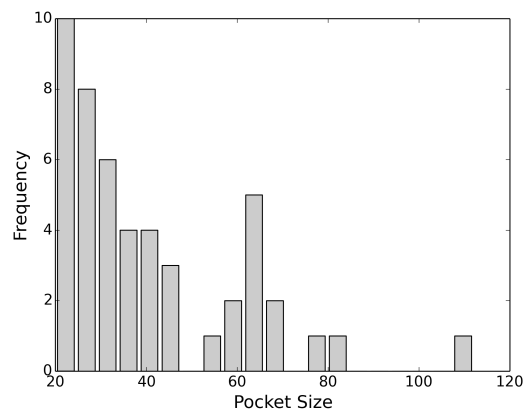

(a) Pocket Size

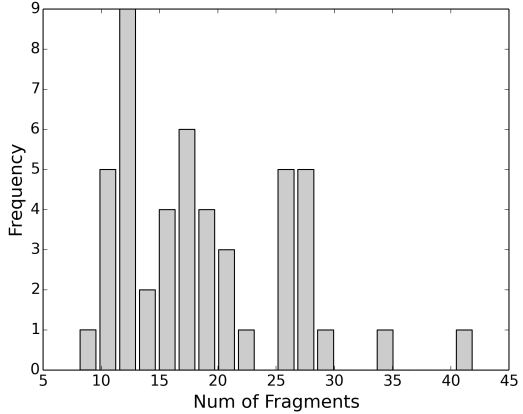

(b) Number of Fragments

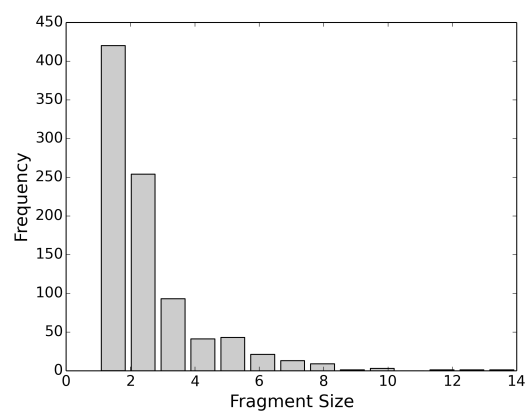

(c) Fragment Size

Figure 4: Statistics of the 48 selected protein pockets with at least 20 residues: (a) most protein pockets contain less than 50 residues; (b) most protein pockets contain a large number of backbone fragments; (c) most backbone fragments on protein pockets contain a small number of residues; and these properties make the the protein structure model superposition problem even more challenging for protein pockets because existing methods rely on a sufficient number of backbone residue fragments.

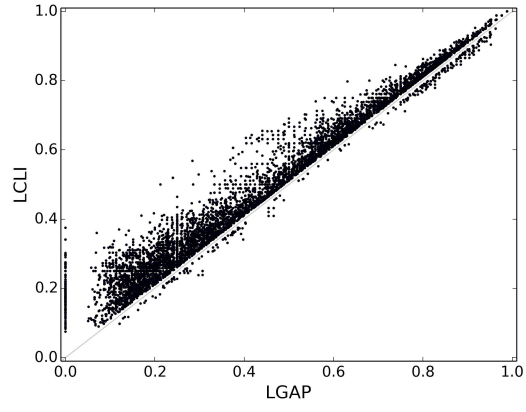

(a) LGAP v.s. LCLI

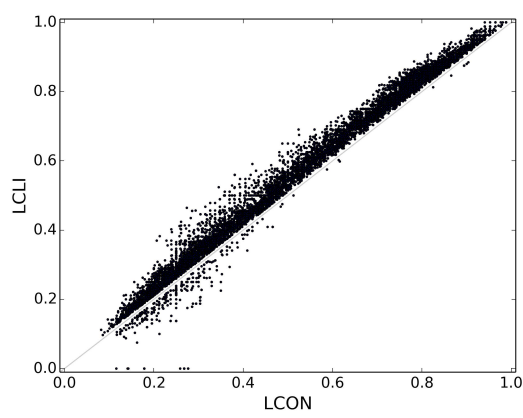

(d) LCON v.s. LCLI

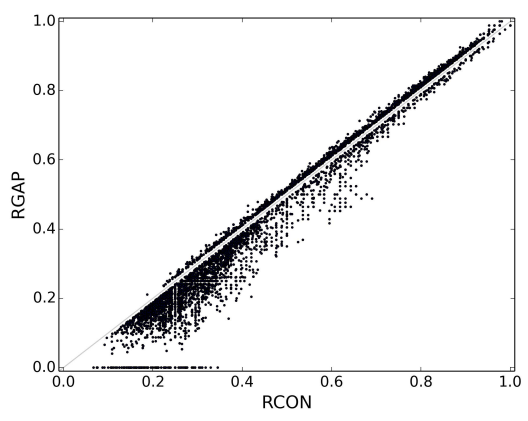

(b) RCON v.s. RGAP

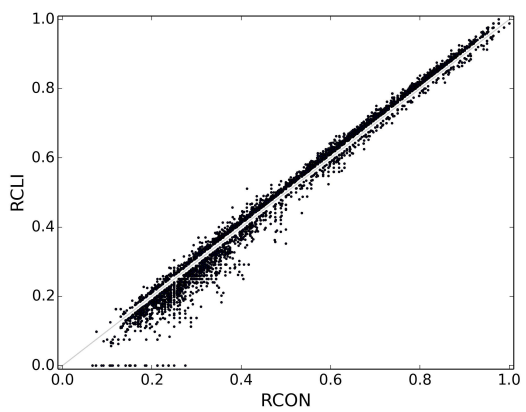

(e) RCON v.s. RCLI

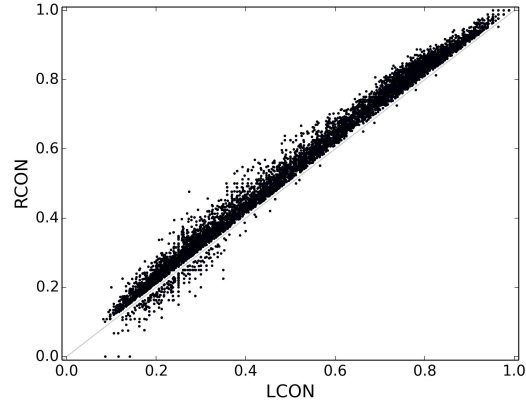

(c) LCON v.s. RCON

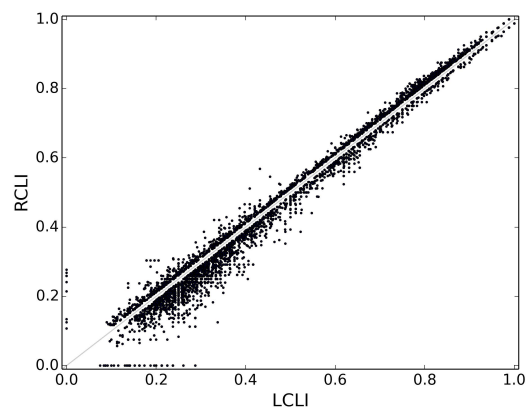

(f) LCLI v.s. RCLI

Figure 5: Comparisons of the GDT-TS scores calculated by the initial superposition sampling with the six types of subsets of corresponding residue pairs defined in Section 2.1 (only cases with alignment score differences over 0.01 are shown): (a,d) local clique subsets are still the best performed local subsets; (b,e) remote consecutive subsets become the new best performed remote subsets, especially for protein pocket models with low similarities; (c,f) remote subsets do not always outperform local subsets any more; and these new observations are the outcomes of the small size and the high fragmentation of protein pockets.

and pocket residues are highly fragmented on protein backbones.

First, we evaluate the performance of each type of subsets of corresponding residue pairs. Consistent with global model superpositions, local clique subsets are the best performed local subsets while the advantages of local clique subsets are more significant for pocket model superpositions, as shown in Figures 5(a) and 5(d). Since most protein pockets contain a small number of residues and the number of remote subsets is the least impacted for remote consecutive subsets, remote consecutive subsets shown in Figures 5(b) and 5(e) become the new best choice among remote subsets. Recall that, for global model superpositions, remote subsets always outperform local subsets. However, Figure 5(f) shows that local clique subsets outperform remote clique subsets. Again, this observation should be caused by the reduced number of re- 


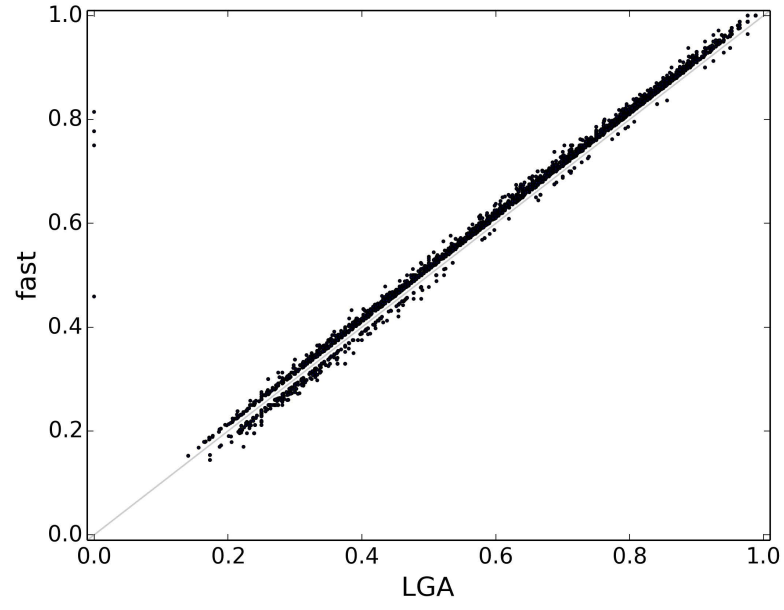

(a) LGA v.s. PROSTA-super (fast version)

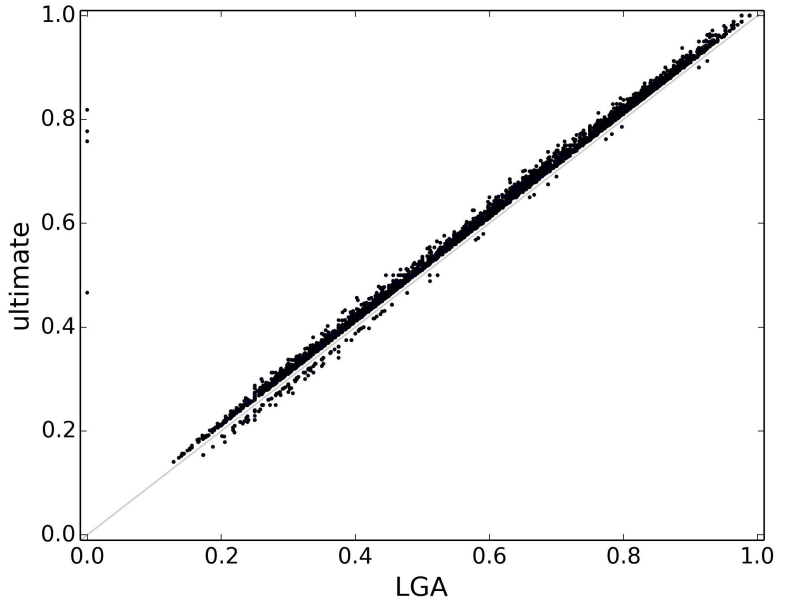

(b) LGA v.s. PROSTA-super (ultimate version)

Figure 6: Comparisons of the GDT-TS scores calculated by PROSTA-super and LGA (only cases with alignment score differences over 0.01 are shown): (a) for the fast version of PROSTA-super, 33.61\% of the alignment scores calculated by PROSTA-super are noticeably higher (i.e., at least 0.01 higher) while $4.39 \%$ of the alignment scores calculated by LGA are noticeably higher; (b) for the ultimate version of PROSTAsuper, $51.82 \%$ of the alignment scores calculated by PROSTA-super are noticeably higher while $1.50 \%$ of the alignment scores calculated by LGA are noticeably higher; and comparing to Figure 3, the advantage of PROSTA-super becomes even bigger for the more challenging protein pocket model superpositions.

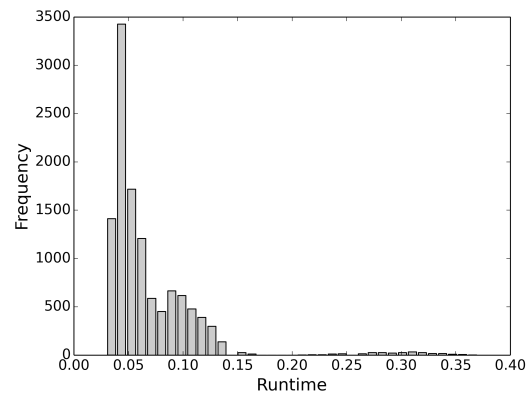

(a) LGA

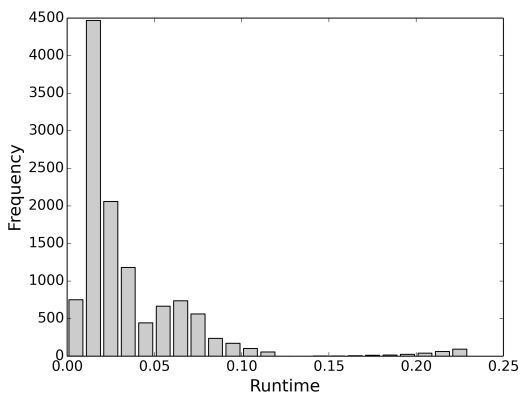

(b) PROSTA-super (fast version)

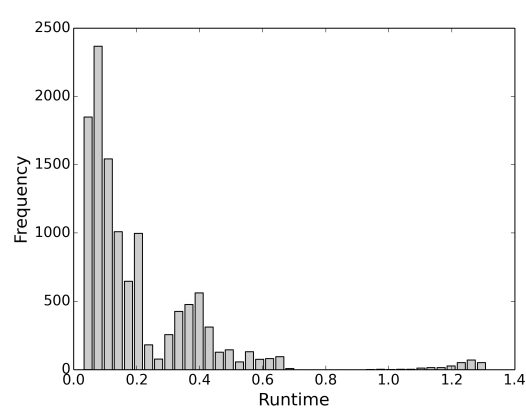

(c) PROSTA-super (ultimate version)

Figure 7: Runtime distributions of LGA and PROSTA-super: (a-c) the average runtime is $0.066 \mathrm{~s}, 0.031 \mathrm{~s}$ and $0.207 \mathrm{~s}$, respectively; and thus the fast version of PROSTA-super produces significantly higher alignment scores while reduces the computational time by approximately half.

mote clique subsets in small pockets. Therefore, we suggest to consider local clique subsets and remote consecutive subsets first for superimposing protein pocket models.

Next, we evaluate the performance of our refinement method with randomly selected subsets of corresponding residue pairs. Due to the space limitations, the results are not included in this article. The biggest observation here is that it is taking significantly higher number of refinement iterations until a near-optimal alignment score is reached. Specifically, it is not rare to observe alignment score improvements after 1,000 refinement iterations. This observation also supports our claim that it is more challenging to optimally superimpose local protein pocket models than global protein structure models. Therefore, we suggest to consider more refinement iterations whenever computational cost is not an issue. Actually, as shown in Figure 7, our implementation of PROSTA-super is very fast, and the computational cost should not be an issue for most users.

Finally, the performance of our protein structure model superposition method is compared to that of LGA [31]. Again, two variants of our PROSTA-super method are evaluated by specifying two different settings. The fast version incorporates local clique subsets, remote consecutive subsets and 50 random subset refinement iterations. As a result, $33.61 \%$ of the alignment scores calculated by PROSTA-super are noticeably higher (i.e., at least 0.01 higher) while $4.39 \%$ of the alignment scores calculated by LGA are noticeably higher. On the other hand, the ultimate version incorporates local and remote consecutive subsets, local and remote clique subsets and 400 random subset refinement iterations. As a result, $51.82 \%$ of the alignment scores calculated by PROSTAsuper are noticeably higher while $1.50 \%$ of the alignment scores calculated by LGA are noticeably higher. Therefore, 
the advantage of our protein structure model superposition method is even bigger for more challenging protein pocket model superpositions.

\section{CONCLUSION}

We have introduced PROSTA-super, a simple and reliable method to optimally superimpose two protein structure models. Importantly, six types of small subsets of corresponding residue pairs are defined based on consecutive and gapped backbone residues and residue contact cliques. Typically, the new clique subsets are more biologically meaningful because they are defined on residue-residue contacts that are not limited to backbone peptide bonds. These clique subsets address the issue of relying on a sufficient number of backbone residue fragments for existing methods, and thus our method is more suitable for superimposing the highly fragmented and small protein pockets.

As a result, the GDT-TS scores [32] calculated by PROSTAsuper are significantly higher than those calculated by LGA [31] while the computational time is reduced by approximately half. Actually, the advantage of PROSTA-super is even bigger for the more challenging local protein pocket model superpositions than those for the classic global protein structure model superpositions. Moreover, the performance advantages of each type of subsets have been demonstrated in this study, and the results could guide end users to choose the right types of subsets for the desired tasks.

\section{Acknowledgments}

The research reported in this publication was supported by competitive research funding from King Abdullah University of Science and Technology (KAUST).

\section{REFERENCES}

[1] H. M. Berman, J. Westbrook, Z. Feng, G. Gilliland, T. N. Bhat, H. Weissig, I. N. Shindyalov, and P. E. Bourne. The protein data bank. Nucleic Acids Research, 28:235-242, 2000.

[2] G. R. Bowman, X. Huang, and V. S. Pande. Using generalized ensemble simulations and markov state models to identify conformational states. Methods, 49(2):197-201, 2009.

[3] X. Cui, S. C. Li, D. Bu, and M. Li. Towards reliable automatic protein structure alignment. In $W A B I$, pages 18-32, 2013.

[4] X. Cui, H. Naveed, and X. Gao. Finding optimal interaction interface alignments between biological complexes. Bioinformatics, 31(12):i133-i141, 2015.

[5] J. Dundas, L. Adamian, and J. Liang. Structural signatures of enzyme binding pockets from order-independent surface alignment: a study of metalloendopeptidase and nad binding proteins. Journal of Molecular Biology, 406(5):713-729, 2011.

[6] X. Gao, D. Bu, S. C. Li, J. Xu, and M. Li. FragQA: predicting local fragment quality of a sequence-structure alignment. Genome Inform, 19:27-39, 2007.

[7] X. Gao, J. Xu, S. C. Li, and M. Li. Predicting local quality of a sequence-structure alignment. J Bioinform Comput Biol, 7(5):789-810, Oct 2009.
[8] J. Janin, R. P. Bahadur, and P. Chakrabarti. Protein-protein interaction and quaternary structure. Quarterly Reviews of Biophysics, 41(02):133-180, 2008.

[9] W. Kabsch. A solution for the best rotation to relate two sets of vectors. Acta Crystallographica Section A: Crystal Physics, Diffraction, Theoretical and General Crystallography, 32(5):922-923, 1976.

[10] L. Kinch, S. Yong Shi, Q. Cong, H. Cheng, Y. Liao, and N. V. Grishin. Casp9 assessment of free modeling target predictions. Proteins, 79(S10):59-73, 2011.

[11] E. Krissinel and K. Henrick. Secondary-structure matching (ssm), a new tool for fast protein structure alignment in three dimensions. Acta Crystallographica Section D: Biological Crystallography, 60(12):2256-2268, 2004.

[12] A. Kryshtafovych, B. Monastyrskyy, and K. Fidelis. Casp prediction center infrastructure and evaluation measures in casp10 and casp roll. Proteins, 2013.

[13] A. T. Laurie and R. M. Jackson. Q-sitefinder: an energy-based method for the prediction of protein-ligand binding sites. Bioinformatics, 21(9):1908-1916, 2005.

[14] J. Liang, C. Woodward, and H. Edelsbrunner. Anatomy of protein pockets and cavities: measurement of binding site geometry and implications for ligand design. Protein Science, $7(9): 1884-1897,1998$.

[15] M. Maadooliat, X. Gao, and J. Z. Huang. Assessing protein conformational sampling methods based on bivariate lag-distributions of backbone angles. Brief Bioinform, 14(6):724-736, Nov 2013.

[16] V. Mariani, F. Kiefer, T. Schmidt, J. Haas, and T. Schwede. Assessment of template based protein structure predictions in casp9. Proteins, 79(S10):37-58, 2011.

[17] K. Olechnovič, E. Kulberkytè, and Č. Venclovas. Cad-score: A new contact area difference-based function for evaluation of protein structural models. Proteins, 81(1):149-162, 2013.

[18] S. Pérot, O. Sperandio, M. A. Miteva, A.-C. Camproux, and B. O. Villoutreix. Druggable pockets and binding site centric chemical space: a paradigm shift in drug discovery. Drug Discovery Today, 15(15):656-667, 2010.

[19] B. Rost. Enzyme function less conserved than anticipated. Journal of Molecular Biology, 318(2):595-608, 2002.

[20] S. Salem, M. J. Zaki, and C. Bystroff. Flexsnap: Flexible non-sequential protein structure alignment. Algorithms for Molecular Biology, 5(1):12, 2010.

[21] N. Siew, A. Elofsson, L. Rychlewski, and D. Fischer. Maxsub: an automated measure for the assessment of protein structure prediction quality. Bioinformatics, 16(9):776-785, 2000.

[22] J. Skolnick and M. Gao. Interplay of physics and evolution in the likely origin of protein biochemical function. Proceedings of the National Academy of Sciences, 110(23):9344-9349, 2013.

[23] D. L. Theobald. Rapid calculation of rmsds using a quaternion-based characteristic polynomial. Acta 
Crystallographica Section A: Foundations of Crystallography, 61(4):478-480, 2005.

[24] A. E. Todd, C. A. Orengo, and J. M. Thornton. Evolution of function in protein superfamilies, from a structural perspective. Journal of Molecular Biology, 307(4):1113-1143, 2001.

[25] S. Wang, J. Ma, J. Peng, and J. Xu. Protein structure alignment beyond spatial proximity. Scientific Reports, 3:1-7, 2013.

[26] Z. Wang, J. Eickholt, and J. Cheng. Multicom: a multi-level combination approach to protein structure prediction and its assessments in casp8. Bioinformatics, 26(7):882-888, 2010.

[27] M. Weisel, E. Proschak, J. M. Kriegl, and G. Schneider. Form follows function: Shape analysis of protein cavities for receptor-based drug design. Proteomics, 9(2):451-459, 2009.

[28] C. A. Wilson, J. Kreychman, and M. Gerstein. Assessing annotation transfer for genomics: quantifying the relations between protein sequence, structure and function through traditional and probabilistic scores. Journal of Molecular Biology, 297(1):233-249, 2000.

[29] L. Xie and P. E. Bourne. Detecting evolutionary relationships across existing fold space, using sequence order-independent profile-profile alignments. Proceedings of the National Academy of sciences, 105(14):5441-5446, 2008.

[30] Y. Yang, J. Zhan, H. Zhao, and Y. Zhou. A new size-independent score for pairwise protein structure alignment and its application to structure classification and nucleic-acid binding prediction. Proteins, 80(8):2080-2088, 2012.

[31] A. Zemla. Lga: a method for finding $3 \mathrm{~d}$ similarities in protein structures. Nucleic Acids Research, 31(13):3370-3374, 2003.

[32] A. Zemla, Č. Venclovas, J. Moult, and K. Fidelis. Processing and analysis of casp3 protein structure predictions. Proteins, 37(S3):22-29, 1999.

[33] J. Zhang and D. Xu. Fast algorithm for population-based protein structural model analysis. Proteomics, 13(2):221-229, 2013.

[34] Y. Zhang and J. Skolnick. Scoring function for automated assessment of protein structure template quality. Proteins, 57(4):702-710, 2004.

[35] Y. Zhang and J. Skolnick. Tm-align: a protein structure alignment algorithm based on the tm-score. Nucleic Acids Research, 33(7):2302-2309, 2005.

[36] D. W. Zimmerman. Teacher's corner: A note on interpretation of the paired-samples t test. Journal of Educational and Behavioral Statistics, 22(3):349-360, 1997. 